\title{
Purple Grape Juice
}

National Cancer Institute

\section{Source}

National Cancer Institute. Purple Grape Juice. NCI Thesaurus. Code C90536.

Juice made form dark, purple grapes that contain polyphenols with antioxidant and

potential cardiovascular protecting activities. Purple grape juice contains high amounts of flavonoids that may increase antioxidant activity and reduce oxidative stress, reduce lowdensity lipoprotein (LDL), and improve nitric oxide formation, endothelial function and vasodilation. 